Pacific Journal of Mathematics

PSEUDOCOMPACT GROUPS 


\title{
PSEUDOCOMPACT GROUPS
}

\author{
HOWARD J. WILCOX
}

This paper is divided into three sections. The first of these concerns itself with extending a result known for compact Abelian topological groups to arbitrary compact topological groups. The particular result is Theorem 1 of "Extensions of Haar Measure for Compact Connected Abelian Groups" by Gerald L. Itzkowitz (Bull. Am. Math. Soc., Vol 71, p. 152-156, 1965). The method used to extend this result is the following: the result is shown to hold for two special cases (when $G$ is a compact $o$-dimensional group; and when $G$ is a product of connected, compact, metric groups), and then it is proven that whenever the result holds for a closed normal subgroup $J$ of a compact group $G$ and also for the factor groups $G / J$ then the result holds for $G$ itself. Using this fact together with the special cases and two known structure theorems yields the desired extension to arbitrary compact groups.

Section II uses the key result of $\S$ I (that many compact groups contain dense pseudocompact subgroups of small cardinality) to show that many Abelian compact groups have infinite compact subgroups which meet the dense pseudocompact subgroups mentioned above in only the identity element. A related result shows that many compact Abelian groups contain dense pseudocompact subgroups which are not countably compact. Counterexamples demonstrate that these results do not hold in general for arbitrary compact groups.

In § III we define the subset of metric elements of a locally compact group to be all those elements which have metric groups as the smallest closed subgroups containing them. We show that for infinite compact Abelian groups the collection $M$ of metric elements is a dense pseudocompact subgroup. We also produce necessary and sufficient conditions for every element of a compact Abelian group to be a metric element. Finally, we show by counterexample that the collection $M$ is not even a subgroup in general when $G$ is nonAbelian.

A topological space is said to be pseudocompact if every continuous real-valued function on the space is bounded. The purpose of this paper is to deal with some properties of pseudocompact and compact topological groups. Throughout the paper $|G|$ will denote the cardinality of a group $G, w(G)$ will denote the weight of $G$ (the least cardinal of a base of open sets for the topology of $G$ ), and $G^{\wedge}$ will denote the character group of $G$. Also, $A\left(G^{\wedge}, H\right)=\left\{\chi \in G^{\wedge}: \chi(x)=1\right.$ for all $x$ in $H\}$ will be called the annihilator of $H$ where $H$ is a 
subgroup of $G$. Our basic reference for known facts will be [4].

In $\S I$ we extend Itzkowitz's Theorem 2 (see [6]) to the case in which $G$ is an arbitrary compact group. We remark at the end of $\S I$ that our method of extending results for compact Abelian groups to the nonAbelian case may be easily applied to other similar problems.

In $\S$ II we obtain some amusing results (for compact Abelian groups) and counterexamples (for nonAbelian groups) which deal with the existence of disjoint pseudocompact and compact subgroups of compact groups.

In $\S$ III we show that the collection $M$ of metric elements (those elements for which the smallest closed subgroup containing them is metric) of a compact Abelian group $G$ is a dense pseudocompact subgroup of $G$. We also show by counterexample that the collection $M$ is not necessarily a subgroup of $G$ if $G$ is not Abelian.

I. Pseudocompact subgroups of compact groups. Throughout this section we will be concerned with generating pseudocompact subgroups of compact groups. We are aided in this endeavor by the following theorem of Comfort and Ross.

THEOREM 1.1. (1.2 of [1]). Let $G$ be a totally bounded group and let $\bar{G}$ be the Weil completion of $G$. If $\mathscr{N}=\{N: N$ is a closed normal subgroup of $\bar{G}$ and $N$ is a $G_{\delta}$-set in $\left.\bar{G}\right\}$, then $G$ is pseudocompact if and only if each translate (in $\bar{G}$ ) of each element $\mathscr{N}$ meets $G$ nontrivially. Also, $G$ is pseudocompact if and only if $G$ meets every nonvoid closed $G_{\delta}$-subset of $\bar{G}$ nontrivially.

We will first show that arbitrary compact topological groups with $w(G)=2^{n}$ (where $n$ is infinite) contain dense pseudocompact subgroups of cardinality $\leqq n^{\aleph_{0}}$. By 1.1 above, this pursuit reduces to that of finding a subset of $G$ with cardinality $\leqq n^{\aleph_{0}}$ which meets (nontrivially) every nonvoid closed $G_{\delta}$-set in $G$. The subgroup generated by this set will have the desired properties. In the Abelian case the result follows easily from a theorem of Itzkowitz.

THEOREM 1.2. (Theorem 2 of [6]). Let $G$ be a compact Abelian topological group satisfying $w(G)=2^{n}$ for some infinite cardinal number $n$. Then $G$ contains a dense pseudocompact subgroup $H$ such that $|H|=n^{\text {No }}$.

THEOREM 1.3. If $G$ is a compact Abelian group with $w(G) \leqq 2^{n}$ where $n$ is infinite, then $G$ contains a dense pseudocompact subgroup $H$ of cardinality $\leqq n^{\aleph_{0}}$. 
Proof. If $w(G)=2^{n}$, we can use 1.2. If $w(G)<2^{n}$, consider the product of $G$ with $2^{n}$ factors of the two element group $\{-1,1\}$. It is easy to show that the weight of this product is $2^{n}$. By 1.2 , the product contains a dense pseudocompact subgroup of cardinality $n{ }^{\aleph_{0}}$. That $G$ contains such a pseudocompact subgroup follows from the easy:

THEOREM 1.4. If $f: G \rightarrow G^{\prime}$ is a continuous mapping of the group $G$ onto the group $G^{\prime}$, and if the set $S$ meets each nonvoid closed $G_{\delta}$-set in $G$, then $f(S)$ meets each nonvoid closed $G_{\delta}$-set in $G^{\prime}$.

1.5 Our problem now is to extend the result in 1.3 to the nonAbelian case. It turns out that proving the result for two special cases (for $G=\prod_{\alpha \epsilon_{A}} G_{\alpha}$ where each $G_{\alpha}$ is a compact connected metric group; and for $G$ compact and o-dimensional) suffices provided that whenever the result holds for a closed normal subgroup $J$ and for $G / J$ then it holds for $G$.

We proceed to prove the first special case (i. e. that in which $G$ is the product mentioned in 1.5). The following lemma will be useful.

LEMMA 1.6. (Lemma 1 of [6]). Let $A$ be any set such that $|A|=2^{n}$ for $n \geqq \aleph_{0}$; then there exists a family $O$ of sequences $\left\{B_{i}\right\}_{i=1}^{\infty}$ of disjoint subsets of $A$ such that

(i) $|\mathscr{O}| \leqq n^{\aleph_{0}}$,

(ii) for any sequence of distinct elements $\left\{\alpha_{i}\right\}_{i=1}^{\infty}$ in $A$, there exists a sequence $\left\{B_{i}\right\}_{i=1}^{\infty}$ in $\bigcirc$ such that $\alpha_{i} \in B_{i}$ for each $i$.

REMARK 1.7. As mentioned previously, we wish to obtain a set $S$ of cardinality $\leqq n^{\aleph_{0}}$ which meets (nontrivially) each nonvoid closed $G_{\delta}$-set in $G$. It is clear that we need consider only $G_{\delta}$-sets which are intersections of basic open sets in $G$; for if point $p \in \bigcap_{i=1}^{\infty} U_{i}$ where each $U_{i}$ is open in $G$, then $p \in \bigcap_{i=1}^{\infty} B_{i}$ where each $B_{i}$ is a basic open set in $G$. We will call $G_{\delta}$-sets of the form $\bigcap_{i=1}^{\infty} B_{i}$ basic $G_{\delta}$-sets.

THEOREM 1.8. If $G$ is an infinite compact group with $w(G) \leqq 2^{n}$ $\left(n \geqq \aleph_{0}\right)$ and if $G=\prod_{\alpha \in A} M_{\alpha}$ where each $M_{\alpha}$ is a compact metric group, then $G$ contains a pseudocompact subgroup $H$ such that $\bar{H}=G$ and $|H| \leqq n$.

Proof. By the remarks in 1.7 we may assume that $w(G)>n$. It is easy to show that $w(G)=|A|$, so that $n<|A| \leqq 2^{n}$ by the above assumption. It is also easy to show that we may assume that each $M_{\alpha}$ is infinite (in fact, each $M_{\alpha}$ has cardinality $c$ ).

(i) Suppose first that $|A|=2^{n}$. Let $\Omega$ be the smallest ordinal number of cardinality $c$, and well order each $M_{\alpha}$ according to $\Omega$ :

$$
M_{\alpha}=\left\{x_{\beta}^{\alpha}: 1 \leqq \beta<\alpha\right\} .
$$


Let $\mathcal{O}$ be as in 1.6. Now for each countable collection (allowing repetitions) $\left\{\gamma_{1}, \gamma_{2}, \cdots\right) \subseteq\{\beta: \beta<\Omega\}$ and for each disjoint sequence $\left(B_{i}\right)_{i=1}^{\infty}$ in $\mathcal{O}$, define

$$
\left(x_{\left\{\gamma_{1}, \gamma_{2}, \ldots\right\} ;\left(B_{i}\right)}\right)_{\alpha}=\left\{\begin{array}{c}
e^{\alpha} \text { if } \alpha \notin \bigcup_{i=1}^{\infty} B_{i} \\
x_{\gamma_{1}}^{\alpha} \text { if } \alpha \in B_{i},
\end{array}\right\}
$$

where $e^{\alpha}$ is the identity in $M_{\alpha}$.

Claim. The collection $X$ consisting of all points $\left(x_{\left\{\gamma_{1}, \gamma_{2}, \ldots\right] ;\left(B_{i}\right)}\right)$ meets each nonvoid $G_{\delta}$ in $G$.

Proof. It is clear that in a product space each "basic $G_{\delta}$ " (see 1.7) depends on at most countably many coordinates.

Let $E$ be such a nonvoid basic $G_{\delta}$ set in $G$, and let $\left\{\alpha_{k}\right\}_{k=1}^{\infty}$ be the set of coordinates on which $E$ is restricted. By 1.6 there exists a disjoint sequence $\left(B_{i}\right)_{i=1}^{\infty}$ in $\mathcal{O}$ such that $\alpha_{k} \in B_{k}$ for all $k$.

Now let $x$ be any element in $E$. Clearly $x_{\alpha_{k}}=x_{\gamma_{i_{k}}}^{\alpha_{k}}$ for some $x_{\gamma_{i_{k}}}$ in the well ordering of $M_{\alpha_{k}}$. Thus $\left(x_{\left.\left\{\gamma_{1}, \gamma_{2} \ldots\right\}_{;}\left(B_{i}\right)_{k=1}^{\infty}\right)}\right.$ meets $E$ and is contained in $X$. This proves the claim.

We complete the proof by noting that $|X| \leqq n^{\aleph_{0}}$ since there are exactly $2^{\aleph_{0}}$ countable collections in $\{\beta: \beta<\Omega\}$ and at most $n^{\aleph_{0}}$ disjoint sequences $\left(B_{i}\right)$ in $\mathcal{O}$ by 1.6 . Thus the group generated by $X$ will have the desired properties by 1.1 .

(ii) If $n<|A|<2^{n}$, choose some factor $M_{\alpha_{n}}$ of $G$ and adjoin $\Pi_{\beta \epsilon_{B}}\left(M_{\alpha_{n}}\right)_{B}=F$ to $G$, where $|B|=2^{n}$.

Now in $G \times F,|A|=2^{n}$, so by (i) above $G \times F$ contains a pseudocompact subgroup $H$ of cardinality $\leqq n^{\aleph_{0}}$ which meets each $G_{\delta}$ in $G \times F$. The projection of $H$ on $G$ will be pseudocompact, dense in $G$, and of cardinality $\leqq n^{\aleph_{0}}$ by 1.1 and 1.4. This completes the proof of 1.8 .

To deal with the second special case (when $G$ is compact and 0 dimensional) we use the following trivial generalization of a result of Kakutani for Abelian groups (see [7]).

THEOREM 1.9. (Kakutani). Let $w(e)$ be the smallest cardinal of a base of open sets at $e$. Then for any compact infinite group $G$, $w(e)=w(G)$.

We now easily prove:

THEOREM 1.10. If $G$ is a compact o-dimensional group and if $w(G) \leqq 2^{n}$ for $n$ infinite, then $G$ contains a dense pseudocompact subgroup of cardinality $\leqq n^{\aleph_{0}}$. 
Proof. By 9.15 of [4], $G$ is homeomorphic with $\{-1,1\}^{w(e)}$. By 1.9 , this is the same as $\{-1,1\}^{w(\theta)}$. By $1.3,\{-1,1\}^{w(G)}$ (which is Abelian) contains a pseudocompact subgroup with the desired properties. By $1.4, G$ contains a subset $S$ which meets every closed $G_{\delta}$-set of $G$. The group generated by $S$ is the desired subgroup of $G$. This completes the proof.

Having dealt with two special cases, we need only prove that if a closed normal subgroup $J$ of $G$ and the factor group $G / J$ both contain dense pseudocompact subgroups of cardinality $\leqq n^{\aleph_{0}}$, then $G$ itself contains a dense pseudocompact subgroup of cardinality $\leqq n^{\aleph_{0}}$. To do this we need the following lemma.

LEMMA 1.11. Let $G$ be a compact group and let $J$ be a closed normal subgroup of $G$. Let $\pi$ be the projection mapping of $G$ onto $G / J$. Then if $E$ is a nonvoid $G_{\delta}$ in $G, \pi(E)$ contains a nonvoid $G_{\delta}$ in $G / J$.

Proof. It is well known that each nonvoid $G_{\delta}$ in a completely regular space contains a nonvoid Baire set. Let $E$ contain the nonvoid Baire set $F$. By 64.G of [3], there exists a compact normal subgroup $N$ of $G$ such that $F \cdot N=F$ and $G / N$ is metrizable.

Consider the following factor groups: $(G / J) /(N \cdot J / J) ; G /(N \cdot J)$; $(G / N) /(N \cdot J / N)$, and note that they are all topologically isomorphic by the second isomorphism theorem for topological groups (see 5.35 of $[4])$.

Claim 1. $\pi(F)=\pi(F) \cdot(N J / J)$ in $G / J$.

Proof. This fact is easy to prove.

Claim 2. $N J / J$ is a $G_{\delta}$-set in $G / J$.

Proof. Since $G / N$ is metrizable, it follows that $(G / N) /(N J / N)$ is metrizable by 8.14 (d) of [4] provided $J N / N$ is closed in $G / N$. But $J$ and $N$ are both compact, so $J \cdot N$ is compact in $G$. Hence $J N / N$ is compact and closed in $G / N$.

Now $(G / N) /(N J / N) \cong G / J N \cong(G / J) /(J N / J)$ are metrizable so that $J N / J$ being a point of $(G / J) /(N J / J)$ is a $G_{\delta}$ in $(G / J) /(J N / J)$. This means that the inverse image of $J N / J$ under the projection map of $G / J$ onto $(G / J) /(J N / J)$ is $N J / J$ which is a $G_{\delta}$ in $G / J$. This proves Claim 2.

By Claim $1, \pi(F)=\pi(F) \cdot(N J / J)$ so that $\pi(F) \subseteq \pi(E)$ contains a translate of $N J / J$, which is a $G_{\delta}$ by Claim 2. This completes the proof of 1.11 .

We can now prove the key theorem. 
THEOREM 1.12. Let $G$ be a compact group with $J$ a closed normal subgroup of $G$. If $J$ and $G / J$ both contain dense pseudocompact subgroups of cardinality $\leqq n^{\aleph_{0}}$, then $G$ contains a pseudocompact subgroup $H$ such that $\bar{H}=G$ and $|H| \leqq n^{\aleph_{0}}$.

Proof. Let $\pi$ be the projection map of $G$ onto $G / J$. Let $F$ be a nonvoid $G_{\delta}$ in $G$. Then by $1.11, \pi(F)$ contains a nonvoid $G_{\delta}$ set in $G / J$.

Let $\{b J: b \in B\}$ be pseudocompact, dense, and of cardinal $\leqq n^{\aleph_{0}}$ in $G / J$. Then $\pi(F)=\{f J: f \in F\}$ contains an element of the form $b J$ for some $b \in B$. Thus $b J \cap F \neq \varnothing$ and $J \cap b^{-1} F \neq \varnothing$.

Let $A$ be pseudocompact, dense and of cardinal $\leqq n^{\aleph_{0}}$ in $J$. Then $A \cap b^{-1} F \neq \varnothing$ since $J \cap b^{-1} F$ is a nonvoid $G_{\delta}$ set in $J$. It follows that $B \cdot A \cap F \neq \varnothing$. Thus the group $H$ generated by $B \cdot A$ is the desired dense pseudocompact subgroup. (Note that if $J$ or $G / J$ itself has cardinality $\leqq n^{\aleph_{0}}$, then $A$ or $\{b J: b \in B\}$ respectively can be taken to be all of $J$ or $G / J$ respectively.) This completes the proof.

We have now obtained the two special cases and the key theorem. In 1.5 we claimed that these results would be sufficient to extend 1.3 to arbitrary compact groups. To see that this is true we introduce the following essential structure theorem.

Theorem 1.13. (Varopoulos in [10]). If $G$ is a connected compact group and $Z$ is its center, then $G / Z$ has the form $\prod_{\alpha \in_{A}} M_{\alpha}$ with all $M_{\alpha}$ compact metrizable groups.

We can now easily obtain:

THEOREM 1.14. If $G$ is an infinite connected compact group with $w(G) \leqq 2^{n}$, then $G$ contains a pseudocompact subgroup $H$ such that $\bar{H}=G$ and $|H| \leqq n^{\aleph_{0}}$.

Proof. If $G$ is Abelian, we are through by 1.3.

If $G$ is not Abelian, consider the center $Z$ of $G$ and the factor group $G / Z$ (note that $Z$ is closed and normal). Clearly $w(Z) \leqq 2^{n}$ and $w(G / Z) \leqq 2^{n}$.

By 1.3, $Z$ contains a pseudocompact subgroup $H_{Z}$ such that $\bar{H}_{Z}=Z$ and $\left|H_{Z}\right| \leqq n^{\aleph_{0}}$. By $1.13, G / Z=\prod_{\alpha \in_{A}} M_{\alpha}$ where each $M_{\alpha}$ is a metric group. By 1.8, $G / Z$ contains a pseudocompact subgroup $H_{G \mid Z}$ such that $\bar{H}_{G / Z}=G / Z$ and $\left|H_{G / Z}\right| \leqq n^{\aleph_{0}}$.

By 1.12, $G$ contains the desired subgroup. This completes the proof of 1.14 .

THEOREM 1.15. If $G$ is an infinite compact group and $w(G) \leqq 2^{n}$, 
then there exists a subgroup $H$ of $G$ which is pseudocompact and such that $\bar{H}=G$ and $|H| \leqq n^{\aleph_{0}}$.

Proof. If $G$ is connected, we are through by 1.14 .

If $G$ is not connected, consider the component $C$ of the identity in $G$ and the factor group $G / C$. Note that $C$ is closed and normal in $G$ by 7.1 of [4]. Clearly both $w(C)$ and $w(G / C)$ are $\leqq 2^{n}$. By 1.14, $C$ contains a pseudocompact subgroup $H_{c}$ such that $H_{c}=C$ and $\left|H_{c}\right| \leqq n^{\aleph_{0}}$. By 7.3 of [4], $G / C$ is o-dimensional; so by $1.10, G / C$ contains a pseudocompact subgroup $H_{\theta / \sigma}$ such that $\bar{H}_{\theta / \sigma}=G / C$ and $\left|H_{G / \sigma}\right| \leqq n^{\aleph_{0}}$.

By 1.12, $G$ contains the desired subgroup $H$. This completes the proof.

REMARK 1.16. It would perhaps be desirable to replace “ $w(G) \leqq$

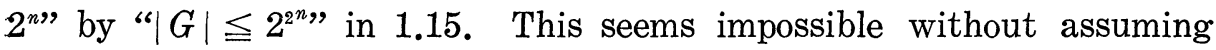
the generalized continuum hypothesis. It is, however, possible to prove that $w(G)=2^{n}$ implies that $|G|=2^{2^{n}}$ without assuming the continuum hypothesis. It is a special case of the following result of Hulanicki.

THEOREM 1.17. (Hulanicki in [5]). If $G$ is $2^{\mathcal{O}(e)}$-compact, then $|G|=2^{\mathscr{O}(e)}$; where $\mathcal{O}(e)$ is the least cardinal of a collection $\left\{U_{i}\right\}$ of open sets such that $\bigcap_{i \in I} U_{i}=\{e\}$ and where "G is $2^{\mathcal{O}(e)}$-compact" means that $G$ is the union of $2^{\mathcal{O}(e)}$ compact sets.

Note that $\mathcal{O}(e)$ in the above theorem is equal to $w(e)$ by the following easy generalization of 8.5 of [4].

THEOREM 1.18. If $G$ is a compact topological group, then $\{e\}=$ $\cap_{\alpha \epsilon_{A}} U_{\alpha}$ where each $U_{\alpha}$ is open in $G$ if and only if $G$ has a basis of open sets of cardinal $|A|$ at $e$.

Thus from 1.17 and 1.18 we see that $w(G)=2^{n}$ implies that $|G|=2^{2 n}$. The converse statement is true if we assume the generalized continuum hypothesis. Thus, assuming the generalized continuum hypothesis, every in infinite compact group of cardinality $2^{2^{n}}$ contains a dense pseudocompact subgroup of cardinality $\leqq n^{\aleph_{0}}$.

REMARK 1.19. It is unfortunate that for certain infinite cardinal numbers $n, n^{\aleph_{0}}>n$. In such instances the pseudocompact subgroup in 1.15 will not be as small in cardinality as might be desired.

It should be pointed out that prior to our knowledge of Hulanicki's result in 1.17, we proved in a manner different from Hulanicki that $w(G)=n$ implies that $|G|=2^{n}$ for arbitrary compact groups. The 
result for compact Abelian groups was proved by Kakutani in [7]. Our proof for the nonAbelian case is completely parallel to the method used earlier to extend 1.3 to arbitrary compact groups. We conjecture that other results known for compact Abelian groups can be extended in like manner to the nonAbelian case.

\section{Disjoint pseudocompact and compact subgroups of compact} groups.

2.1 In view of Theorem 1.15, it is reasonable to ask (1) whether a compact group $G$ with $w(G) \leqq 2^{n}$ which contains a dense pseudocompact subgroup $H$ of cardinality $\leqq n^{\aleph_{0}}$ also contains an infinite compact subgroup $F$ such that $F \cap H=\{e\}$.

It may also be asked: (2) given $H$ of cardinality $\leqq n^{\aleph_{0}}$, dense and pseudocompact in $G$, whether we can find another dense pseudocompact subgroup $J$ of $G$ such that $J \cap H=\{e\}$.

It turns out that in the Abelian case the answer to the first question is usually (with certain cardinality restrictions) yes. The second question is seen to generally have a negative answer by an example in which a pseudocompact subgroup of a compact Abelian group $G$ is seen to meet every other dense pseudocompact subgroup nontrivially.

The example cited above also shows us that if we first choose the infinite compact subgroup $F$ of question (1) and attempt to find a pseudocompact subgroup $H$ such that $H \cap F=\{e\}$ then we will probably be unsuccessful. The example shows a two-element compact subgroup which meets every dense pseudocompact subgroup in $G$.

We first answer question (1).

THEOREM 2.2. If $G$ is a compact Abelian group with $w(G)=2^{n}$ for some cardinal $n \geqq \boldsymbol{\aleph}_{0}$, and if $n^{\boldsymbol{\aleph}_{0}}<2^{n}$, then given any dense pseudocompact subgroup $H$ of cardinality $\leqq n^{\aleph_{0}}$, there exists an infinite compact subgroup $F$ of $G$ such that $F \cap H=\{e\} . \quad$ (Note that such groups $H$ exist by 1.15.)

Proof. By 1.17 and hypothesis, $|H|<w(G)<|G|$. For each $x \in H, x \neq e$, choose $\chi_{x} \in G^{\wedge}$ with the propetry that $\chi_{x}(x) \neq 1$ (this is possible by 22.17 of [4]).

Now define $\mathscr{H}$ to be the subgroup of $G^{\wedge}$ generated by the set $\left\{\chi_{x}: x \in H\right\}$. Clearly $|\mathscr{H}| \leqq|H|<\left|G^{\wedge}\right|=w(G)$ so that $G^{\wedge} / \mathscr{H}$ is infinite. It follows that $A(\mathscr{H})=\{x \in G: \chi(x)=1$ for every $\chi \in \mathscr{H}\}$ is a closed (hence compact) infinite subgroup of $G$; it is isomorphic with the character group of $G^{\wedge} / \mathscr{H}$ (see 23.24 and 23.25 of [4]). 
Call $A(\mathscr{H})=F$.

Now if $y \in H \cap F$ and $y \neq e$, then there exists $\chi_{y} \in \mathscr{H}$ with the property that $\chi_{y}(y) \neq 1$. But $\chi_{y}(y)=1$ since $y \in F$. Thus $y=e$ and $H \cap F=\{e\}$. This completes the proof.

In [8] Kister provides an example of a pseudocompact, noncountably compact subgroup of a compact group $G$. We now show that such subgroups are a rather common occurrence.

THEOREM 2.3. If $G$ is a compact Abelian group with $w(G)=2^{n}$ for some cardinal $n \geqq \aleph_{0}$ and $n^{\aleph_{0}}<2^{n}$, then $G$ contains a pseudocompact, noncountably compact subgroup $K$.

Proof. By 2.2 and 1.15, $G$ contains a pseudocompact subgroup and an infinite compact subgroup ( $H$ and $F$ respectively) such that $H \cap F=\{e\}$. By 2.5 (2) of [9], $F$ contains an infinite metric subgroup $M$. Now $M$ is second countable and hence separable. Let $J$ be a dense countable subgroup of $M$, and let $\left\{x_{n}\right\}_{n=1}^{\infty}$ be a sequence in $J$ converging to $s \in M-J$.

Consider the group $K=H \cdot J$. It is pseudocompact since it contains $H$; but it is not countably compact since $\left\{x_{n}\right\}_{n=1}^{\infty}$ is contained in $K$, but $s \notin K$. This completes the proof.

COROLlaRY 2.4. If $G, H$, and $K$ are as in 2.3, then there exists an infinite compact group $F^{\prime}$ such that $F^{\prime} \cap K=\{e\}$.

Proof. Observe that $|K|=|H|$ since $J \cdot H=K$ and $J$ is countable. Apply the proof of 2.2 with $K$ replacing $H$. This completes the proof.

We now show that 2.2 was true because we first chose the pseudocompact subgroup $H$ and then found the disjoint infinite compact subgroup $F$.

EXAMPLE 2.5. Let $G=\Pi_{b \in_{B}}\{-1,1\}_{b} \times\{-1, i, 1,-i\}$ where $|B|>\aleph_{0}$. Then $G$ is a compact subgroup of $T^{|B|}$ where $T$ is the circle group.

Let $F$ be the two-element compact subgroup $F=\Pi_{b \in_{B}}\{1\}_{b} \times\{1,-1\}$ of $G$. (Note that $w(G)=|B|$.)

Claim. Any pseudocompact subgroup $H$ of $G$ meets $F$ nontrivially.

Proof. Observe that $E=\Pi_{b \in_{B}}\{-1,1\}_{b} \times\{i\}$ is a $G_{\delta}$-set of $G$. By 1.1, any pseudocompact subgroup $H$ which is dense in $G$ meets $E$. But $x \in E \cap H$ implies that $x \cdot x \in F \cap H$, and $x \cdot x \neq e$. This proves 
the claim.

2.6 We now use the same example to show that question (2) in 2.1 has a negative answer.

Let $G$ and $E$ be as in 2.5. Let $H$ and $K$ be any dense pseudocompact subgroups of $G$; then there exists $h \in H \cap E$ and $k \in K \cap E$. Hence $h^{2} \in H, k^{2} \in K$, and yet $h^{2}=k^{2}=\prod_{b \in_{B}}\{1\}_{b} \times i^{2} \neq e$. So the dense pseudocompact subgroups $H$ and $K$ are not disjoint.

2.7 At this point we note that all of the above results required commutativity of the groups involved. The results for the nonAbelian case have been evasive thus far.

\section{The subgroup $M$ of metric elements of $G$.}

3.1 We now turn to a slightly different pursuit. Until now we have been content to produce pseudocompact subgroups of compact groups by choosing points from certain $G_{\delta}$ subsets of the compact group and using them to generate the desired pseudocompact subgroup. We will now supply a more concrete example of a dense pseudocompact subgroup of a compact Abelian group $G$.

We begin with the following definition.

Definition 3.2. Let $G$ be a locally compact group and let $x \in G$. Denote by $H_{x}$ the smallest closed subgroup of $G$ containing $x$. Then

(i) If $H_{x}$ is metrizable, then $x$ will be called a metric element of $G$ (note that if $H_{x}$ is finite, then $x$ is usually called torsion).

The collection of metric elements of $G$ will be denoted by $M$. We will show that if $G$ is a compact Abelian group then its subset of metric elements is a dense pseudocompact subgroup of $G$.

We begin with the following lemma.

LEMMA 3.3. Let $f$ be a continuous homomorphism of the compact group $G$ onto the compact group $K$. Then $f(x)$ is a metric element of $K$ whenever $x$ is a metric element in $G$.

Proof. Let $x$ be a metric element in $G$. Then $H_{x}$ is a compact metric group. We will show that $f\left(H_{x}\right)$ is a metric group which implies that $H_{f(x)}$ is a metric group.

Let $J=\left\{y \in H_{x}: f(y)=e\right\}$. Then $J$ is closed and $J=\bigcap_{n=1}^{\infty} U_{n}$ for some sequence $\left\{U_{n}\right\}$ of open sets in $H_{x}$. Now $H_{x}-J=H_{x}-\bigcap_{n=1}^{\infty} U_{n}=$ $\bigcup_{n=1}^{\infty}\left(H_{x}-U_{n}\right)$, and $f\left(H_{x}-J\right)=\bigcup_{n=1}^{\infty} f\left(H_{x}-U_{n}\right)$. Thus $f\left(H_{x}\right)-$ $f\left(H_{x}-J\right)=f\left(H_{x}\right)-\bigcup_{n=1}^{\infty} f\left(H_{x}-U_{n}\right)=\bigcap_{n=1}^{\infty}\left(f\left(H_{x}\right)-f\left(H_{x}-U_{n}\right)\right)$ which is a $G_{\delta}$ set in $f\left(H_{x}\right)$. But $f\left(H_{x}\right)-f\left(H_{x}-J\right)=\{e)$, so by 8.3 of [4] 
$f\left(H_{x}\right)$ is a (compact) metric group. This completes the proof.

THEOREM 3.4. If $G$ is a compact Abelian group, then the collection $M$ of metric elements of $G$ is a subgroup of $G$.

Proof. In 3.3 it was actually proved that a continuous (homomorphic) mapping of a compact group $G$ onto a compact group $K$ preserves compact metric subgroups. The theorem follows from this and the fact that the mapping $(a, b) \rightarrow a \cdot b^{-1}$ of $H_{x} \times H_{y} \rightarrow H_{x} \cdot H_{y}{ }^{-1}$ is a continuous mapping. Note that if $G$ is not Abelian then $H_{x} \cdot H_{y}{ }^{-1}$ is not necessarily a group.

We now wish to prove that the subgroup $M$ meets every nonvoid closed $G_{\delta}$ set in $G$. We first prove the following lemma which is a special case.

LEMMA 3.5. Let $G=\prod_{\alpha \in A} M_{\alpha}$ where each $M_{\alpha}$ is a compact metric group. Then $M$ (the set of metric elements of $G$ ) intersects every nonvoid closed $G_{\delta}$ set in $G$.

Proof. The theorem follows easily from the fact that each basic $G_{\delta}$-set (see 1.7) in a product space depends on at most countably many factors of the product and the fact that a countable product of metric groups is a metric group.

We now use some known structure theorems together with 3.5.

THEOREM 3.6. If $G$ is a compact Abelian group and if $M$ is the subgroup of metric elements of $G$, then $M$ is a dense pseudocompact subgroup of $G$.

Proof. Since $G$ is compact, $G^{\wedge}$ is discrete. It is known that any Abelian group is embeddable in a divisible group (see A-15 of [4]) so that $G^{\wedge}$ is embedded in a discrete divisible group $\mathscr{H}$. It is also known (see A-14 of [4]) that $\mathscr{H}$ is topologically isomorphic with $\sum_{\alpha \epsilon_{A}} G_{\alpha}$ where each $G_{\alpha}$ is a countable group. Now $\mathscr{H}^{\wedge}$ is topologically isomorphic with $\Pi_{\alpha \in_{A}} G_{\alpha}^{\wedge}$ where each $G_{\alpha}^{\wedge}$ is a compact metric group by 23.22 of [4]. By 24.11 of [4], $G$ is topologically isomorphic with $\mathscr{H}^{\wedge} / A\left(\mathscr{H}^{\wedge}, G^{\wedge}\right)$. That is, $G$ is a continuous homomorphic image of $\mathscr{H}^{\wedge}$. By 3.5, $M^{\prime}$ (the subgroup of metric elements of $\mathscr{H}^{\wedge}$ ) is dense and pseudocompact in $\mathscr{H}^{\wedge}$. By 3.3 , the continuous homomorphic mapping of $\mathscr{H}^{\wedge}$ to $G$ takes $M^{\prime}$ into $M$. By 1.1 and 1.4, the image of $M^{\prime}$ is dense and pseudocompact in $G$. Thus $M$ is dense and pseudocompact in $G$. This completes the proof.

We next derive some necessary and sufficient conditions for $M$ to 
be all of $G$. We need the following lemmas.

LEMMA 3.7. If $G$ is a 0-dimensional compact group, then $M$ (the subset of metric elements of $G$ ) is all of $G$.

Proof. Let $x \in G$. Then $H_{x}$ is 0 -dimensional and monothetic (i. e. $H_{x}$ is the closure of a group generated by a single element). By 25.16 of [4], $H_{x}$ is topologically isomorphic with a metrizable group. This completes the proof.

LEMMA 3.8. If $G$ is a compact group and if $J$ is a metrizable closed normal subgroup of $G$, then whenever $\mathscr{C}$ is a closed metrizable subgroup of $G / J, \pi^{-1}(\mathscr{H})$ is a closed metrizable subgroup of $G$ (where. $\pi$ is the projection of $G$ on $G / J)$.

Proof. Let $H=\pi^{-1}(\mathscr{H})$. Then $H$ is clearly a closed subgroup. of $G$. By the second isomorphism theorem for topological groups (5.34 of [4]), $H / J$ is topologically isomorphic with $\mathscr{H}$. Thus $H / J$ is metrizable. By hypothesis $J$ is metrizable, so by 8.3 and 5.38 of [4], $H$ is metrizable. This completes the proof.

We now present the desired theorem.

THEOREM 3.9. Let $G$ be a compact Abelian group, $M$ its subgroup of metric elements, $C$ the component of the identity in $G$, and $M_{0}$ the subgroup of metric elements in $C$. Then the following statements are equivalent:

(i) $G=M$,

(ii) $C$ is metrizable,

(iii) $C=M_{c}$.

Proof. That (i) implies (iii) is clear (note that $M_{0}=M \cap C$ ).

To prove that (iii) implies (ii), assume that $C=M_{c}$, but $C$ is not metrizable. Now if $C$ is monothetic (i. e. $H_{x}=C$ for some $x \in C$ ), then $x$ is not a metric element since $C$ is not metric by assumption. But this contradicts $C=M_{c}$, and so (iii) implies (ii) if $C$ is monothetic.

If $C$ is not monothetic, then $w(G)>c$ by 25.14 of [4]. Let $\mathscr{H}$ be a subgroup of cardinality $c$ in $C^{\wedge}$ and consider $A(C, \mathscr{H})$ which is a closed subgroup (see 23.24 of [4]) of $C$. By 24.10 of [4], $\mathscr{C}=$ $A\left(C^{\wedge}, A(C, \mathscr{H})\right)$, so we use 23.25 of [4] with $H=A(C, \mathscr{H})$ to obtain that $\left|(C / H)^{\wedge}\right|$ is $c$. Thus by 25.14 of [4], $C / H$ is monothetic, and since $(C / H)^{\wedge}$ is not countable, $C / H$ is not metrizable. By the proof in the previous paragraph, $C / H$ contains a nonmetric element; and by 3.3, $C$ contains a nonmetric element. That is, $C \neq M_{c}$ which contradicts. 
our initial assumption. Thus (iii) implies (ii).

To prove that (ii) implies (i), assume that $C$ is metrizable. Let $x$ be any element of $G$. We will show that $H_{x}$ is metrizable.

Consider the element $x C$ of the 0-dimensional factor group $G / C$. By $3.7, x C$ belongs to a closed metrizable subgroup $\mathscr{H}$ of $G / C$. Then $H=\pi^{-1}(\mathscr{H})$ is a closed metrizable subgroup of $G$ by 3.8. Since $x \in H, H_{x}$ is also a closed metrizable subgroup of $G$, and $M=G$. This completes the proof of 3.9 .

COROLlaRY 3.10. If $G$ is compact, connected Abelian group, then $G=M$ if and only if $G$ is metric.

We shall now turn to the case in which $G$ is compact and nonAbelian. We would first like to know whether $M$ (the subset of metric elements in $G$ ) is always a subgroup of $G$. We show that such is not the case by the following example.

EXAMPLE 3.11. Let $G$ be the group generated by $a$ and $b$ where $a^{2}=b^{2}=e$. Then $a=a^{-1}, b=b^{-1}$, and $G=\{e, a, a b, a b a, \cdots, b, b a, b a b, \cdots\}$. Let $H$ consist of all elements of even length; that is,

$$
H=\{e, a b, a b a b, \cdots, b a, b a b a, \cdots\} .
$$

Then $H$ is an infinite cyclic group which is normal in $G$ and Abelian. Also, $H$ is isomorphic with $Z$ (the integers) and is of index 2 in $G$.

Let $\mathscr{C}$ be a collection of characters on $H$ which separates points and is uncountable. Let $T_{\mathscr{C}}$ be the topology induced on $H$ by $\mathscr{H}$. Then $\left(H, T_{\mathscr{H}}\right)$ is a totally bounded group by 1.5 of [2].

Let $\mathscr{U}$ be an open basis at $e$ for topology on $H$. Then $\mathscr{C}$ satisfies conditions (i) - (iv) of 4.5 of [4]. Hence $\mathscr{C}$ is a family of subsets of $G$ with the finite intersection property. Clearly (i) - (iii) of 4.5 of [4] hold for the collection $\mathscr{C}$ taken as a collection of subsets of $G$. We show that (iv) also holds on $G$. Let $x \in G$ and $U \in \mathscr{C}$. If $x \in H$, we are through since (iv) holds for $x \in H$. Now note that $a V a^{-1}=a V a=$ $V^{-1}$ and $b V b^{-1}=b V b=V^{-1}$ for $V \in \mathscr{C}$. Now suppose $x \in a H$. Since $a H=\{a, b, b a b$, babab, $\cdots, a b a b a, a b a, \cdots\}$, it follows that $x V x^{-1}=x V x=$ $V^{-1}$ for $V \in \mathscr{W}$. Since for $U \in \mathscr{C}$, there exists a $V \in \mathscr{C}$ such that $V^{-1} \subseteq U$ by (ii), (iv) follows immediately. Hence $\{x U: x \in G, U \in \mathscr{C}\}$ is an open subbase for a topology on $G$. Since $G=H \cup a H$ and $H$ is totally bounded, it follows that $G$ is totally bounded.

Now the Weil completion $\bar{G}$ is a compact topological group containing $a, b$, and $a b$. Clearly $a$ and $b$ are metric elements of $G$; but $a b$ is not a metric element since $H_{a b}=\vec{H}$ which is topologically isomorphic with $\mathscr{H}_{d}$ by 1.3 and 1.12 of [2], and $\bar{H}$ is not metrizable since $\bar{H}^{\wedge} \cong \mathscr{H}_{d}$ which is uncountable. Thus $M$ is not a subgroup of $G$. 
REMARK 3.12. Even though $M$ fails, in general, to be a subgroup of $G$, we may still ask whether the subset $M$ of a compact group $G$ meets every closed $G_{\delta}$ of $G$. This turns out to be true. It follows easily from the Abelian case.

THeOREM 3.13. Let $G$ be a compact group and $M$ its subset of metric elements. Then $M$ meets every nonvoid closed $G_{\delta}$ set in $G$.

Proof. Let $E$ be a nonvoid closed $G_{\delta}$ set in $G$. Then $E \cap H_{x} \neq \varnothing$ for some $x \in G$. Now $H_{x}$ is a compact Abelian group and $E \cap H_{x}$ is a nonvoid closed $G_{\delta}$-set in $H_{x}$; so by 3.6 there is an element $y \in E \cap H_{x}$ such that $H_{y}$ is a closed metric subgroup of $H_{x}$. But $H_{y}$ is also a closed metric subgroup of $G$. Hence $y \in M \cap E$, and $M$ meets every closed $G_{\delta}$ in $G$. This completes the proof.

We now show that part of theorem 3.9 holds for nonAbelian $G$.

LEMMA 3.14. If $J$ is a closed metric subgroup of a compact group $G$, then $M_{G / J}$ (the set of metric elements in $G / J$ ) is equal to. $G / J$ if and only if $M=G$.

Proof. Since $G / J$ is a continuous homomorphic image of $G$, if $G=M$ it follows from 3.3 that $G / J=M_{G / J}$.

By $3.8, \pi^{-1}\left(M_{\theta / J}\right) \leqq M$. If $\pi^{-1}\left(M_{G / J}\right)=\pi^{-1}(G / J)$, then $G=M$. This completes the proof.

THEOREM 3.15. If $G$ is a compact group with $C$ (the component of the identity in $G$ ) metric, then $M=G$.

Proof. By 7.3 of [4], $G / C$ is 0-dimensional and hence $G / C=M_{G / \sigma}$ (the set of metric elements in $G / C$ ) by 3.7. By Lemma 3.14, $G=M$. This completes the proof.

In conclusion we conjecture that converse to 3.15 is true.

\section{BIBLIOGRAPHY}

1. W. W. Comfort and Kenneth A. Ross, Pseudocompactness and uniform continuity in topological groups, Pacific J. Math. 16 (1966), 483-496.

2. - Topologies induced by groups of characters, Fund. Math. 55 (1964), 283291.

3. Paul R. Halmos, Measure theory, D. Van Nostrand Company, Princeton, 1950.

4. Edwin Hewitt and Kenneth A. Ross, Abstract Harmonic Analysis I, SpringerVerlag, Heidelberg, 1963.

5. A. Hulanick, On cardinal numbers related with locally compact groups, Bulletin De L'Academie Polonaise des Sciences VI-2 (1958), 67-70. 
6. Gerald L. Itzkowitz, Extensions of Haar measure for compact connected abelian groups, Bull. Am. Math. Soc. 71 (1965), 152-156.

7. Shizuo Kakutani, On cardinal numbers related with a compact abelian group, Proc. Imp. Acad., Tokyo, 19 (1943), 366-372.

8. J. M. Kister, Uniform continuity and compactness in topological groups, Proc. Am. Math. Soc. 13 (1962), 37-40.

9. Walter Rudin, Fourier Analysis on Groups, Interscience Publishers, 1962.

10. N. Th. Varopoulos, A theorem on the continuity of homomorphisms of locally compact groups, Proc. Camb. Phil. Soc. 60 (1964), 449-463.

Received September 21, 1965, and in revised form January 23, 1966. This paper contains the main results of the author's $\mathrm{Ph}$. D. dissertation (University of Rochester) which was written under the direction of Prof. Kenneth A. Ross.

UNIVERSITY OF CONNECTICUT 



\title{
PACIFIC JOURNAL OF MATHEMATICS
}

\author{
EDITORS
}

\section{H. SAMELSON}

Stanford University

Stanford, California

J. P. JANS

University of Washington

Seattle, Washington 98105
J. DugundJI

University of Southern California

Los Angeles, California 90007

RICHARD ARENS

University of California

Los Angeles, California 90024

\section{ASSOCIATE EDITORS}

\section{E. F. BECKENBACH}

B. H. NEUMANN

F. WOLF

K. YosidA

\section{SUPPORTING INSTITUTIONS}

\author{
UNIVERSITY OF BRITISH COLUMBIA \\ CALIFORNIA INSTITUTE OF TECHNOLOGY \\ UNIVERSITY OF CALIFORNIA \\ MONTANA STATE UNIVERSITY \\ UNIVERSITY OF NEVADA \\ NEW MEXICO STATE UNIVERSITY \\ OREGON STATE UNIVERSITY \\ UNIVERSITY OF OREGON \\ OSAKA UNIVERSITY \\ UNIVERSITY OF SOUTHERN CALIFORNIA
}

\author{
STANFORD UNIVERSITY \\ UNIVERSITY OF TOKYO \\ UNIVERSITY OF UTAH \\ WASHINGTON STATE UNIVERSITY \\ UNIVERSITY OF WASHINGTON \\ AMERICAN MATHEMATICAL SOCIETY \\ CHEVRON RESEARCH CORPORATION \\ TRW SYSTEMS \\ NAVAL ORDNANCE TEST STATION
}

Mathematical papers intended for publication in the Pacific Journal of Mathematics should be typewritten (double spaced). The first paragraph or two must be capable of being used separately as a synopsis of the entire paper. It should not contain references to the bibliography. Manuscripts may be sent to any one of the four editors. All other communications to the editors should be addressed to the managing editor, Richard Arens at the University of California, Los Angeles, California 90024.

50 reprints per author of each article are furnished free of charge; additional copies may be obtained at cost in multiples of 50 .

The Pacific Journal of Mathematics is published monthly. Effective with Volume 16 the price per volume (3 numbers) is $\$ 8.00$; single issues, $\$ 3.00$. Special price for current issues to individual faculty members of supporting institutions and to individual members of the American Mathematical Society: $\$ 4.00$ per volume; single issues $\$ 1.50$. Back numbers are available.

Subscriptions, orders for back numbers, and changes of address should be sent to Pacific Journal of Mathematics, 103 Highland Boulevard, Berkeley 8, California.

Printed at Kokusai Bunken Insatsusha (International Academic Printing Co., Ltd.), No. 6, 2-chome, Fujimi-cho, Chiyoda-ku, Tokyo, Japan.

PUBLISHED BY PACIFIC JOURNAL OF MATHEMATICS, A NON-PROFIT CORPORATION

The Supporting Institutions listed above contribute to the cost of publication of this Journal, but they are not owners or publishers and have no responsibility for its content or policies. 


\section{Pacific Journal of Mathematics \\ Vol. 19, No. $2 \quad$ June, 1966}

Leonard Daniel Baumert, Extreme copositive quadratic forms . ......... 197

Fred James Bellar, Jr., Pointwise bounds for the second initial-boundary value problem of parabolic type ........................ 205

L. Carlitz and David Paul Roselle, Restricted bipartite partitions ........ 221

Robin Ward Chaney, On the transformation of integrals in measure space ........................................... 229

Colin W. Clark, An embedding theorem for function spaces ........... 243

Edwin Duda, A theorem on one-to-one mappings................ 253

Ben Fitzpatrick, Jr. and Donald Reginald Traylor, Two theorems on metrizability of Moore spaces.......................... 259

Allen Roy Freedman, An inequality for the density of the sum of sets of vectors in $n$-dimensional space ....................... 265

Michael Friedberg, On representations of certain semigroups .......... 269

Robert William Gilmer, Jr., The pseudo-radical of a commutative ring . . . . 275

Hikosaburo Komatsu, Fractional powers of operators ............... 285

Daniel Rider, Transformations of Fourier coefficients ................. 347

David Alan Sánchez, Some existence theorems in the calculus of variations ...................................... 357

Howard Joseph Wilcox, Pseudocompact groups............... 365

William P. Ziemer, Some lower bounds for Lebesgue area ............. 381 